${ }^{1}$ Centro de Pacientes Críticos, Clínica las Condes. Santiago, Chile.

2Unidad ECMO, Departamento de Anestesia, Clínica las Condes. Santiago, Chile.

Los autores no declaran conflicto de intereses.

El trabajo no contó con apoyo financiero.

Recibido el 30 de diciembre de 2016, aceptado el 12 de junio de 2017.

Correspondencia a: Dr. Tomás Regueira Estoril 450, Las Condes. tregueira@clinicalascondes.cl

\section{Factibilidad del uso de bivalirudina como anticoagulante en soporte vital extracorpóreo}

\author{
NICOLETTE VAN SINT JAN ${ }^{1}$, RODRIGO DÍAZ ${ }^{2}$, \\ CHRISTIAN FAJARDO ${ }^{2}$, ROCIO AGLIATTI ${ }^{2}$, MARCELA PALAVECINO ${ }^{1}$, \\ PABLO HASBÚN ${ }^{1}$, TOMÁS REGUEIRA ${ }^{1}$
}

\section{Experience with anticoagulation with bivalirudin during extracorporeal membrane oxygenation}

Background: Extracorporeal membrane oxygenation (ECMO) is used with increasing frequency in patients with respiratory and cardiac failure. The achievement of an adequate anticoagulation is critical to avoid patient and circuit complications. Aim: To assess the feasibility and safety of anticoagulation with bivalirudin, as an alternative to unfractionated heparin (UFH), in patient with ECMO. Material and Methods: Observational study, which included all patients receiving anticoagulation with bivalirudin during ECMO, according to a standardized protocol, between august 2015 to May 2016. Results: Bivalirudin was used in 13 out 70 patients connected to ECMO. Ten procedures were for cardiac support and three for respiratory support. Mortality was $43 \%$. ECMO lasted $31 \pm 31$ days. The time of UFH use before changing to bivalirudin was 7 \pm 7 days. The reasons to change to bivalirudin were inadequate levels of partial thromboplastin time (PTT) in nine patients, and heparin induced thrombocytopenia (HIT) in four patients. The time of bivalirudin use was $24 \pm 33$ days. Per patient, a mean of $2.7 \pm 4$ oxygenators were changed. These had a useful life of 11.4 and 19.1 days during UFH and bivalirudin use, respectively. The mean bivalirudin dose was $0.08 \pm 0.04 \mathrm{mg} / \mathrm{kg} / \mathrm{h}$. There was no significant bleeding, thrombosis or circuit obstruction during its use. PTT levels $(p<0.01)$ and platelet count $(p<0.01)$ increased significantly after the start of bivalirudin use in patients with UHF resistance and HIT, respectively. Conclusions: Bivalirudin was a safe and efficient drug for anticoagulation during ECMO. It is important to have an alternative drug for anticoagulation in ECMO patients.

(Rev Med Chile 2017; 145: 710-715)

Key words: Anticoagulants; Antithrombins; Extracorporeal Membrane Oxygenation; Heparin.
$\mathrm{E}$ 1 soporte vital extracorpóreo (ECMO, del inglés extracorporeal membrane oxigenation) es utilizado cada vez con mayor frecuencia y éxito, tanto en patologías respiratoria como cardíaca ${ }^{1,2}$. Los criterios de ingreso son cada vez más flexibles en términos de gravedad y condición de base de los pacientes, lo que se asocia a procesos de soporte de cada vez mayor duración.
Lograr una anticoagulación efectiva es fundamental durante el tiempo en ECMO. Una anticoagulación inefectiva se asocia a embolias sistémicas, hemorragia, pérdidas de volumen progresivas $o$ agudas del circuito y alteraciones de la membrana de oxigenación, todas estas complicaciones se asocian a riesgo de muerte.

La droga más comúnmente usada para lograr 
este fin es la heparina no fraccionada (HNF). La HNF actúa uniéndose a antitrombina para, en conjunto, inactivar trombina, factor $\mathrm{X}$ activado, entre otros ${ }^{3}$. La HNF es el anticoagulante de elección por ser fácilmente titulable, fácil de medir su efecto, de bajo costo y por tener un antídoto que permite la reversión de su efecto en pocos minutos.

Sin embargo, en ciertas situaciones no es posible utilizar HNF en forma adecuada o prolongada. Entre las causas más frecuentes para buscar alternativas de anticoagulación en ECMO a la HNF están la sospecha de trombocitopenia inducida por heparina (HIT, del inglés heparin induced thrombocytopenia $)^{4}$, la resistencia a heparina secundaria a déficit de antitrombina III (ATIII) ${ }^{5} \mathrm{o}$ la imposibilidad de lograr niveles adecuados de anticoagulación con HNF.

La bivalirudina es un inhibidor selectivo directo de la trombina. Inhibe de forma reversible el lugar catalítico de la trombina, neutralizando los efectos de esta, es decir, interfiere con la formación de fibrina, la agregación plaquetaria y la activación del factor XII. A diferencia de la heparina, la bivalirudina no requiere antitrombina III para su activación ${ }^{6}$. Al unirse reversiblemente a la trombina, los efectos anticoagulantes de la bivalirudina desaparecen poco después de su interrupción. Su uso se ha estudiado ampliamente en el contexto de revascularización miocárdica ${ }^{7}$, pero no existen estudios clínicos con distribución aleatoria o comparativos respecto a su uso en ECMO.

En este estudio se busca observar la factibilidad y seguridad del uso de bivalirudina como alternativa a la HNF en pacientes en ECMO.

\section{Métodos}

Estudio descriptivo, no intervencional, que incluye todos los pacientes en ECMO, tanto de origen respiratorio como cardiovascular, anticoagulados con bivalirudina entre agosto de 2015 y mayo de 2016.

\section{Protocolo de anticoagulación}

Todos los pacientes fueron anticoagulados de acuerdo a protocolo de la unidad para pacientes en ECMO. Brevemente, todo paciente que inicia una terapia de soporte vital extracorpóreo, y que no tiene contraindicación para anticoagulación, inicia HNF. Se utilizan 50 a 100 U/Kg para canulación con control posterior de TTPK. Luego se inicia HNF de mantención por vía exclusiva o preoxigenador a $10 \mathrm{U} / \mathrm{Kg} / \mathrm{h}$ y se ajusta según TTPK.

En pacientes con déficit documentado de ATIII, sospecha de HIT, o bien con imposibilidad de lograr meta de TTPK luego de 3 controles consecutivos, el equipo clínico considera el cambio a bivalirudina.

\section{Dosis de bivalirudina}

Se comienza infusión a $0,05 \mathrm{mg} / \mathrm{kg} / \mathrm{h}$ sin bolo, se ajusta según TTPK, y se controla inicialmente a las $3 \mathrm{~h}$ y luego cada $6 \mathrm{~h}$, según protocolo.

\section{Estadística}

El presente estudio constituye un estudio preliminar de factibilidad, de naturaleza descriptiva. La comparación de variables se realizó con test de Friedmann. Para el análisis de cambios en el comportamiento de las diferentes variables estudiadas se consideraron los 3 días previos al inicio a bivalirudina (paciente en HNF) y los 6 días posteriores al inicio de bivalirudina. Se consideró una $\mathrm{p}<0,05$ como significativa. Se ocupó el programa SPSS v15.0. El estudio fue aprobado por el Comité de Ética de Clínica Las Condes.

\section{Resultados}

Se usó bivalirudina en 13 de 70 pacientes que se conectaron a ECMO en el período estudiado. De estos, 7 fueron mujeres y 6 hombres. El promedio de edad fue de $33 \pm 25$ años, siendo 3 pacientes pediátricos y 10 adultos.

Respecto del tipo de ECMO, tres correspondieron a soporte primariamente respiratorio y 10 para soporte de la función cardíaca. De los 13 pacientes que recibieron bivalirudina como anticoagulante, 11 pacientes lograron salir de ECMO y 2 fallecieron durante el soporte; la mortalidad intrahospitalaria global fue de $42,9 \%$.

Los días de ventilación mecánica pre-ECMO promedio fue 4,7 \pm 9 días; y el promedio de días en ECMO fue de 31,2 \pm 31 días, es decir, en general fueron soportes de larga duración. La oxigenación, medida por $\mathrm{PaFi}$, al ingreso a ECMO respiratorio fue de $85,5 \pm 53 \mathrm{mmHg}$.

Respecto al paso de HNF a bivalirudina, los días promedio entre el inicio de HNF a cambio a bivalirudina fueron $7 \pm 7$ días. Las causas de 
cambio a bivalirudina fueron la imposibilidad de lograr niveles de TTPK suficientes en 9 pacientes, y la sospecha de trombocitopenia inducida por heparina en 4 pacientes. El promedio de días con bivalirudina fue de $24 \pm 33$ días.

El promedio de cambio de oxigenadores fue de $2,7 \pm 4$ por paciente, con una vida útil por filtro de 11,4 días para la heparina y 19,1 días para bivalirudina. Ningún paciente presentó episodios de sangrado u obstrucciones agudas del circuito significativos durante el uso de bivalirudina.

La dosis promedio de bivalirudina en todos los pacientes fue de $0,08 \pm 0,04 \mathrm{mg} / \mathrm{kg} / \mathrm{h}$. Los niveles de TTPK aumentaron significativamente una vez iniciada bivalirudina $(\mathrm{p}=0,014)$ en el total de los pacientes y en forma aun más significativa en los pacientes con resistencia a heparina o con varialidad excesiva de TTPK en HNF $(\mathrm{p}<0,001)$ (Figura 1).

Los niveles de plaquetas aumentaron significativamente en aquellos pacientes con sospecha de HIT $(\mathrm{p}=0,006)$ (Figura 2) una vez iniciada la infusión de bivalirudina. Los niveles de hemoglobina $(\mathrm{p}=0,3)$, de LDH $(\mathrm{p}=0,5)$ y de fibrinógeno $(\mathrm{p}=0,5)$ se mantuvieron estables en el total de pacientes. El inicio de bivalirudina se asoció
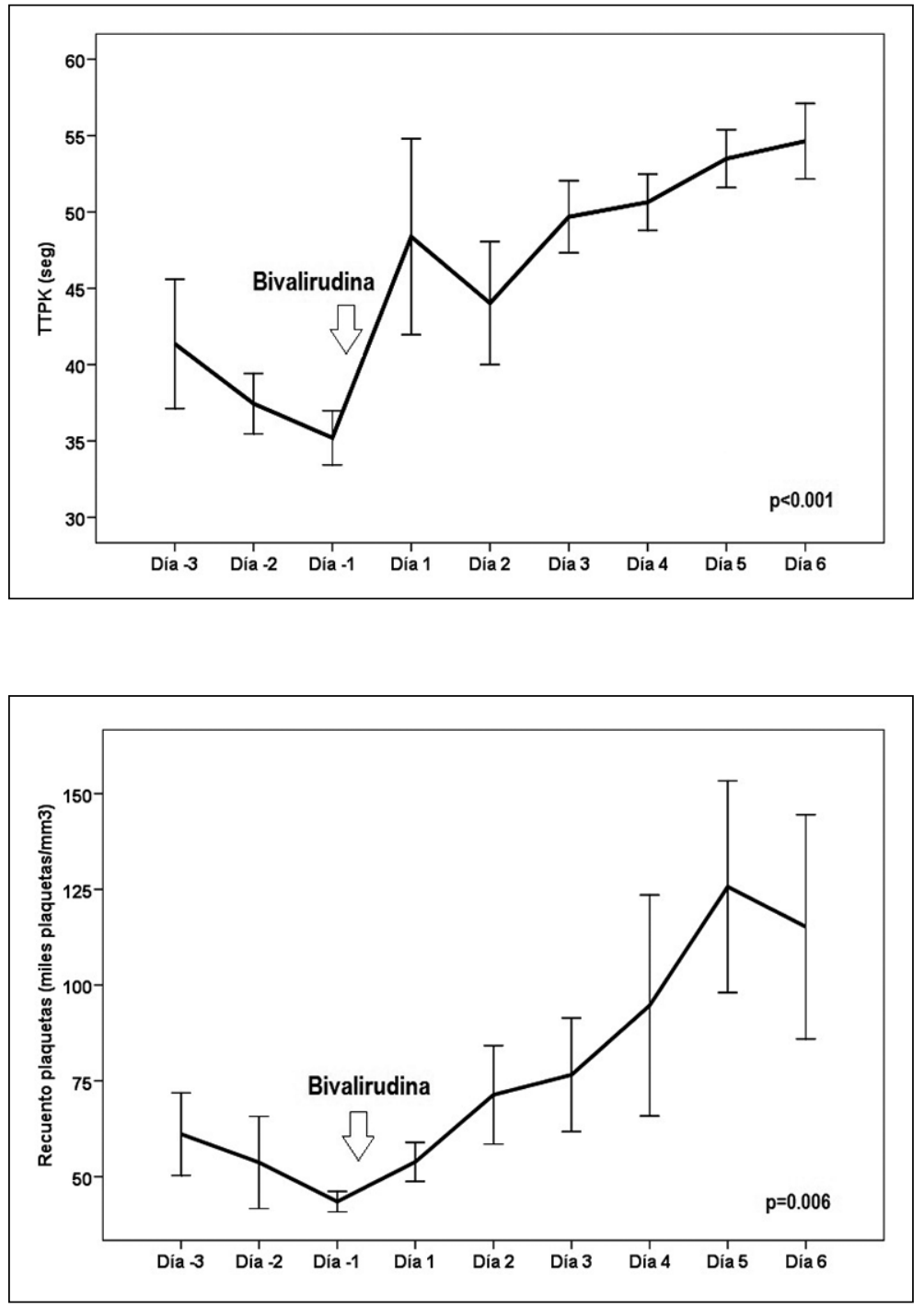

Figura 1. Evolución de los valores del tiempo parcial de tromboplastina (TTPK) los 3 días previos al inicio de bivalirudina y los 6 días posteriores. Estadistica: test de Friedman.
Figura 2. Evolución de los recuentos de plaquetas los 3 días previos al inicio de bivalirudina y los 6 días posteriores. Estadística: test de Friedman. 
Tabla 1. Parámetros relevantes en la evolución de los pacientes

\begin{tabular}{|c|c|c|c|c|c|c|c|c|c|}
\hline Variable & Día -3 & Día -2 & Día -1 & \multirow{5}{*}{ 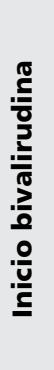 } & Día 1 & Día 2 & Día 3 & Día 4 & Valor $\mathbf{p}$ \\
\hline $\begin{array}{l}\mathrm{LDH} \\
(\mathrm{UI} / \mathrm{I})\end{array}$ & $827 \pm 650$ & $789 \pm 580$ & $665 \pm 362$ & & $637 \pm 397$ & $639 \pm 315$ & $634 \pm 311$ & $743 \pm 375$ & NS \\
\hline $\begin{array}{l}\text { Hemoglobina } \\
(g / d l)\end{array}$ & $10,0 \pm 1,6$ & $10,0 \pm 1$ & $10,7 \pm 1,6$ & & $10,2 \pm 1,8$ & $10,3 \pm 1,4$ & $10,4 \pm 1,7$ & $10,0 \pm 1,6$ & NS \\
\hline $\begin{array}{l}\text { Fibrinógeno } \\
(\mathrm{mg} / \mathrm{dl})\end{array}$ & $201 \pm 100$ & $259 \pm 220$ & $349 \pm 230$ & & $355 \pm 213$ & $360 \pm 208$ & $317 \pm 145$ & $353 \pm 151$ & NS \\
\hline $\begin{array}{l}\text { Bilirrubina } \\
(\mathrm{mg} / \mathrm{dl})\end{array}$ & $2,68 \pm 2$ & $2,59 \pm 2$ & $3,37 \pm 2$ & & $3,47 \pm 3$ & $2,84 \pm 2$ & $1,94 \pm 2$ & $2,05 \pm 2$ & 0,006 \\
\hline
\end{tabular}

LDH: lactato deshidrogenasa. Valor p: Friedmann test.

a una disminución significativa de los niveles plasmáticos de bilirrubina en todos los pacientes $(p=0,006)$, y en particular, en aquellos con resistencia a heparina o con variabilidad excesiva de TTPK en HNF $(\mathrm{p}=0,046)$ (Tabla 1$)$.

\section{Conclusiones}

En esta serie de casos clínicos observamos que $18 \%$ de los pacientes en ECMO no logran anticoagularse segura y adecuadamente con HNF. Las causas observadas fueron la dificultad de lograr niveles de anticoagulación suficientes o estables o bien la aparición de trombocitopenia. Esto es concordante con lo reportado previamente en la literatura ${ }^{8-10}$ y subraya la importancia de contar con alternativas a la HNF para lograr anticoagulación adecuada en ECMO.

La bivalirudina se comportó como una droga segura y eficiente en lograr niveles de anticoagulación adecuados en todos los pacientes. Coincidentemente, aquellos pacientes en los cuales se cambió la anticoagulación desde HNF a bivalirudina por sospecha de HIT aumentaron significativamente su recuento plaquetario, así como los pacientes con dificultad para anticoagular aumentaron significativamente sus niveles de TTPK.

En 9 pacientes no fue posible lograr niveles adecuados de anticoagulación con HNF, fenómeno conocido como resistencia a heparina y definido por la necesidad de más de $35.000 \mathrm{U}$ en $24 \mathrm{~h}$ para lograr mantener el tiempo parcial de protrombina (TTPK) en rangos terapéuticos ${ }^{11,12}$. La imposibilidad de lograr niveles suficientes de anticoagulación con HNF se debe con mayor frecuencia a déficit de antitrombina III (ATIII), a un aumento en el metabolismo de la HNF, o por el aumento durante la inflamación de proteínas plasmáticas de fase aguda que unen heparina como el fibrinógeno, el factor VIII y el factor plaquetario $4^{13}$. El déficit de ATIII puede revertirse con el uso de plasma fresco congelado (como donante de ATIII) o concentrados de ATIII. Estas soluciones son de alto costo, se asocian a sobrecarga de volumen y a riesgo de reacciones transfusionales. Por lo anterior, el uso de una droga alternativa como bivalirudina es una opción a considerar.

La bivalirudina ha sido estudiada extensamente y es utilizada en el contexto de reperfusión coronaria. La fisiopatología del síndrome coronario agudo (SCA) involucra la activación plaquetaria y la formación de trombo luego de la ruptura de placa, con la consecuente expresión de trombina en las diferentes membranas celulares y plaquetarias. La trombina, a su vez, amplifica la respuesta trombótica. El tratamiento del SCA se basa en el tratamiento antiplaquetario (aspirina, clopidogrel, entre otras) y drogas antitrombínicas, como la HNF, heparinas de bajo peso molecular, o bien, con drogas como la bivalirudina. Un metaanálisis reciente en revascularización coronaria percutánea muestra que el uso de bivalirudina, en comparación con $\mathrm{HNF}$, reduce el riesgo de mortalidad por cualquier causa y los sangrados mayores ${ }^{14}$. Sin embargo, faltan estudios de mayor tamaño para su incorporación en las guías de manejo.

La bivalirudina ha sido empleada preliminarmente en pacientes en $\mathrm{ECMO}^{15-17}$, con resultados favorables, similares a los presentados en este estudio, sin aumento de las complicaciones trombóticas ni hemorrágicas. No existen protocolos de uso 
recomendados para pacientes en ECMO, siendo extrapolados desde la experiencia en cardiología intervencional, de hecho, las dosis utilizadas en este estudio son algo superiores a las reportadas en estudios previos ${ }^{15-17}$. Sin embargo, con las dosis utilizadas, y sin emplear bolo, observamos una alta eficacia para lograr niveles adecuados de anticoagulación, y no se presentaron complicaciones hemorrágicas. Las dosis necesarias para lograr anticoagulación efectiva en ECMO son más bajas que las reportadas para el manejo del síndrome coronario agudo, las cuales consideran uso de bolo $(0,75 \mathrm{mg} / \mathrm{kg})$ e infusiones del orden de $1,75 \mathrm{mg} /$ $\mathrm{kg} / \mathrm{h}^{18,19}$. Por otro lado, sabemos que la correlación entre TTPK y la concentración de bivalirudina es una línea curva de concavidad inferior, pero su uso en las dosis bajas utilizadas, a diferencia de las dosis en CEC, y por la experiencia publicada por otros grupos nos permite utilizar este examen como una medición del efecto de este anticoagulante ${ }^{20}$.

Las limitaciones para el uso de bivalirudina en la actualidad son el costo, el cual es varias veces mayor al de la HNF, y la ausencia de antídoto disponible. Respecto a esto último, aun cuando no siempre predecible, la vida media de la bivalirudina es muy corta, de aproximadamente $25 \mathrm{~min}$, explicada por metabolismo mayoritariamente por proteólisis plasmática, lo que hace que la bivalirudina pueda ser considerada una droga segura, a pesar de no tener un antídoto específico. Por otro lado, bivalirudina tiene un porcentaje de metabolismo renal, por lo que en pacientes con función renal alterada debe monitorizarse adecuadamente en estos pacientes y considerar el riesgo asociado al aumento de su vida media hasta de $1 \mathrm{~h}$ en pacientes con falla renal severa. Considerando lo anterior, y el hecho que en un grupo no menor de pacientes no será posible lograr niveles adecuados de anticoagulación con HNF, o bien, ante la aparición de trombocitopenia, aparece como deseable contar con una droga de alternativa para los pacientes en ECMO, particularmente si el soporte será prolongado.

En conclusión, en una serie limitada de pacientes, el uso de bivalirudina parece ser una alternativa eficiente y segura para los pacientes en ECMO que no pueden seguir usando HNF. Considerando la estabilidad de la anticoagulación lograda con bivalirudina, sería interesante contar con estudios que comparen ambas drogas como elección de anticoagulación inicial.

\section{Referencias}

1. Rozencwajg S, Pilcher D, Combes A, Schmidt M. Outcomes and survival prediction models for severe adult acute respiratory distress syndrome treated with extracorporeal membrane oxygenation. Crit Care 2016; 20 (1): 392-402.

2. Pellegrino V, Hockings LE, Davies A. Veno-arterial extracorporeal membrane oxygenation for adult cardiovascular failure. Curr Opin Crit Care 2014; 20 (5): 484-92.

3. Mulloy B, Hogwood J, Gray E, Lever R, Page CP. Pharmacology of Heparin and Related Drugs. Pharmacol Rev 2016; 68 (1): 76-141.

4. Warkentin TE. Heparin-induced thrombocytopenia. Curr Opin Crit Care 2015; 21 (6): 576-85.

5. Spiess BD. Treating heparin resistance with antithrombin or fresh frozen plasma. Ann Thorac Surg 2008; 85 (6): 2153-60.

6. Lupi A, Rognoni A, Cavallino C, Secco GG, Prando MD, Santagostino M, et al. Pharmacological adjuvant therapies in primary coronary interventions: bivalirudin. Cardiovasc Hematol Agents Med Chem 2013; 11 (2): 106-14.

7. Mahmoud A, Saad M, Elgendy AY, Abuzaid A, Elgendy IY. Bivalirudin in percutaneous coronary intervention, is it the anticoagulant of choice?. Cardiovasc Ther 2015; 33 (4): 227-35.

8. Avidan MS, Levy JH, Scholz J, Delphin E, Rosseel PM, Howie MB, et al. A phase III, double-blind, placebo-controlled, multicenter study on the efficacy of recombinant human antithrombin in heparin-resistant patients scheduled to undergo cardiac surgery necessitating cardiopulmonary bypass. Anesthesiology 2005; 102 (2): 276-84.

9. Hassel K. Heparin-Induced Thrombocytopenia: Diagnosis and Management. Thromb Res 2008; 123 (1): 16-21.

10. Baldwin ZK, Spitzer AL, Ng VL, Harken AH. Contemporary standards for the diagnosis and treatment of heparin-induced thrombocytopenia. Surgery 2008; 143 (3): 305-12.

11. Hirsh J, Warkentin TE, Shaughnessy SG, Anand SS, Halperin JL, Raschke R, et al. Heparin and low-molecular-weight heparin: mechanisms of action, pharmacokinetics, dosing, monitoring, efficacy, and safety. Chest 2001; 119 (1): 64-94.

12. Finley A, Greenberg C. Heparin sensitivity and resistance: management during cardiopulmonary bypass. Anesth Analg 2013; 116 (6): 1210-22.

13. Mousavi S, Moradi M, Khorshidahmad T, Motamedi M. Anti-Inflammatory Effects of Heparin and Its Deri- 
vatives: A Systematic Review. Adv Pharmacol Sci 2015; 507151.

14. Shah R, Rogers KC, Matin K, Askari R, Rao SV. An updated comprehensive meta-analysis of bivalirudin vs heparin use in primary percutaneous coronary intervention. Am Heart J 2016; 171 (1): 14-24.

15. Pieri M, Agracheva N, Bonaveglio E, Greco T, De Bonis $\mathrm{M}$, Covello RD, et al. Bivalirudin versus heparin as an anticoagulant during extracorporeal membrane oxygenation: a case-control study. J Cardiothorac Vasc Anesth 2013; 27 (1): 30-4.

16. Sanfilippo F, Asmussen S, Maybauer DM, Santonocito C, Fraser JF, Erdoes G, et al. Bivalirudin for Alternative Anticoagulation in Extracorporeal Membrane Oxygenation: A Systematic Review. J Intensive Care Med 2016; 29: Epub ahead of print.

17. Jyoti A, Maheshwari A, Daniel E, Motihar A, Bhathiwal
RS, Sharma D. Bivalirudin in venovenous extracorporeal membrane oxygenation. J Extra Corpor Technol 2014; 46 (1): 94-7.

18. Schulz S, Richardt G, Laugwitz KL, Morath T, Neudecker J, Hoppmann P, et al. Bavarian Reperfusion Alternatives Evaluation (BRAVE) 4 Investigators. Prasugrel plus bivalirudin vs. clopidogrel plus heparin in patients with ST-segment elevation myocardial infarction. Eur Heart J 2014; 35 (34): 2285-94.

19. Steg PG, van 't Hof A, Hamm CW, Clemmensen P, Lapostolle F, Coste P, et al. EUROMAX Investigators. Bivalirudin started during emergency transport for primary PCI. N Engl J Med 2013; 369 (23): 2207-17.

20. Burcham PK, Rozycki A, Abel E. Considerations for analgosedation and antithrombotic management during extracorporeal life support. Ann Transl Med 2017; 5 (4): 69. 